\title{
SUPRA-CONSERVATIVE FINITE-VOLUME METHODS FOR THE SIMULATION OF SUBSONIC FLOW
}

\author{
ARTHUR E.P. VELDMAN ${ }^{1}$ \\ ${ }^{1}$ Bernoulli Institute, University of Groningen, \\ P.O. Box 407, 9700AK Groningen, The Netherlands \\ e-mail: a.e.p.veldman@rug.nl - URL: www.math.rug.nl/ veldman
}

Key words: CFD, Conservation Laws, Finite-Volume, Supra-Conservative Discretization

\begin{abstract}
It is demonstrated how finite-volume methods can be designed such that, next to the primary invariants (mass, momentum and internal energy), they also conserve secondary invariants (kinetic energy), i.e., they are supra-conservative. Key ingredient is a consistency between the discrete divergence terms in the constituting equations and the discrete pressure gradient. The requirements hold for any discretization method with a volume-consistent scaling.
\end{abstract}

\section{INTRODUCTION}

It has been found advantageous for discretized flow equations to possess additional (secondary) invariants, next to the (primary) invariants from the constituting conservation laws. Much effort has been put in finding formulations of the flow equations that lead to these additional properties - a recent overview of such attempts for the compressible flow equations is presented in $[1,2]$. Analytically all formulations are equivalent, because of the conservation of mass, and they possess the same invariants. But after discretization this equivalence is partly lost, and differences appear in the induced discrete invariants.

The Euler equations describing fluid dynamics can be expressed as conservation laws in terms of primary variables mass density $\rho$, momentum per unit mass (= velocity) $\boldsymbol{u}$, and internal energy per unit mass $e$ :

$$
\frac{\partial \rho}{\partial t}+\nabla \cdot \boldsymbol{m}=0 ; \quad \frac{\partial \rho \boldsymbol{u}}{\partial t}+\nabla \cdot(\boldsymbol{m} \otimes \boldsymbol{u})=-\nabla p ; \quad \frac{\partial \rho e}{\partial t}+\nabla \cdot(\boldsymbol{m} e)=-p \nabla \cdot \boldsymbol{u} .
$$

Here, $\boldsymbol{m} \equiv \rho \boldsymbol{u}$ denotes the mass flux and $p$ the pressure. The set of equations is closed by an equation of state $p=p(\rho, e)$.

The introduction of the mass flux $\boldsymbol{m}$ will help to distinguish between the two appearances of $\boldsymbol{u}$ in the momentum equation: one as transporting velocity, the other as transported quantity. Most of the considerations in this paper hold for any vector field $\boldsymbol{m}$.

The equations are solved on a (two- or three-dimensional) domain $\Omega$ with appropriate initial and boundary conditions. For convenience, we will assume either homogeneous boundary conditions or periodic ones, in order not to bother with the boundaries. Physically, this means that external influences on the flow field are excluded. 
Background The earliest mention of the favorable influence of preserving discrete kinetic energy can be found around 1960 while studying non-linear instabilities in long-time integration for weather prediction $[3,4]$. An important ingredient is the skew-symmetric discretization of convection [5]. These ideas have led to a follow-up in finite-difference options for incompressible flow on uniform grids as reviewed by Morinishi [6]. A generalization to non-uniform grids was presented in [7] and further generalized to structured curvilinear staggered grids in [8]. Discrete skew-symmetry of the convective terms also features in the summation-by-parts (SBP) method [9].

In the 1990s, inspired by [10], the symmetry-preserving higher-order finite-volume approach of Verstappen and Veldman [11-13] combined discrete mass, momentum and energy conservation for incompressible flow on non-uniform, staggered Cartesian grids. They emphasized the need for, counter-intuitive, geometry-independent interpolations for the fluxes. The generalization to unstructured, collocated and staggered, grids has been presented in $[14,15]$.

For compressible flow, also early use of skew-symmetric forms can be mentioned, such as the formulation by Feiereisen et al. [16]. Consistency between the individual discrete equations is found beneficial for stability $[17,18]$. To numerically describe shock wave discontinuities the use of entropy variables can be profitable $[19,20]$, but often discrete momentum conservation is lost.

In contrast, and complementary, our interest is in the treatment of the relatively smooth (possibly turbulent) part of the flow; hence the restriction to subsonic flow. Yet, due to the absence of numerical diffusion, our approach will not interfere with the, necessarily, dissipative character of numerical shock treatment. We wo
erties and to extend them with additional secc
in this vein can be montioned already, e.g. [21
Next to the spatial discretization, also the symplectic methods $[26,27]$. Further, compres
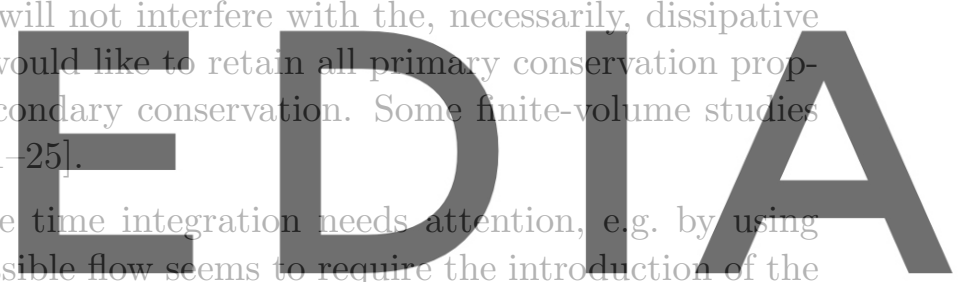

square root of the density $\sqrt{\rho}$, as discussed in [24,28-31]. For an overview of energy-preserving

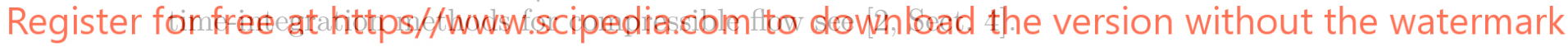

Supra-conservative discretization The general idea behind many of the above methods is to discretely conserve more (secondary) invariants additional to the (primary) ones in the conservation laws. Therefore, these methods are coined supra-conservative and designed according to a principle of non-interference [32]. This requires compatibility between the discrete operators in the equations of motion. We will discuss the details of such a discrete compatibility for structured and unstructured computational grids with a collocated positioning of the unknowns.

In our discussion we will start from the discrete finite-volume formulation of the basic equations (1), and never return to the analytical formulation [33]. In this way discrete conservation of the primary invariants is guaranteed from the start. Then, at the discrete level, the freedom left in the formulation will be used to generate additional properties like secondary invariants. 


\section{CONSERVATION OF ENERGY - ANALYTIC}

The flow equations 'automatically' describe the conservation properties of mass, momentum and internal energy. They additionally convectively preserve total energy by a proper exchange of kinetic and internal energy. The analytic derivation of this property forms a guide line to mimic step-by-step in the discretization.

From the primary conservation laws one can deduce secondary conservation laws for kinetic energy $\rho E_{\text {kin }} \equiv \frac{1}{2} \rho \boldsymbol{u}^{2}$ and total energy $\rho E_{\text {tot }} \equiv \rho\left(E_{\text {kin }}+e\right)$. The evolution of the total energy can be calculated analytically as a weighted combination of the primary conservation laws (1). Equation (2) schematically shows how the derivation of the energy evolution proceeds. It is stressed that this derivation holds for any $\boldsymbol{m}$ : its explicit value $\rho \boldsymbol{u}$ is not used.

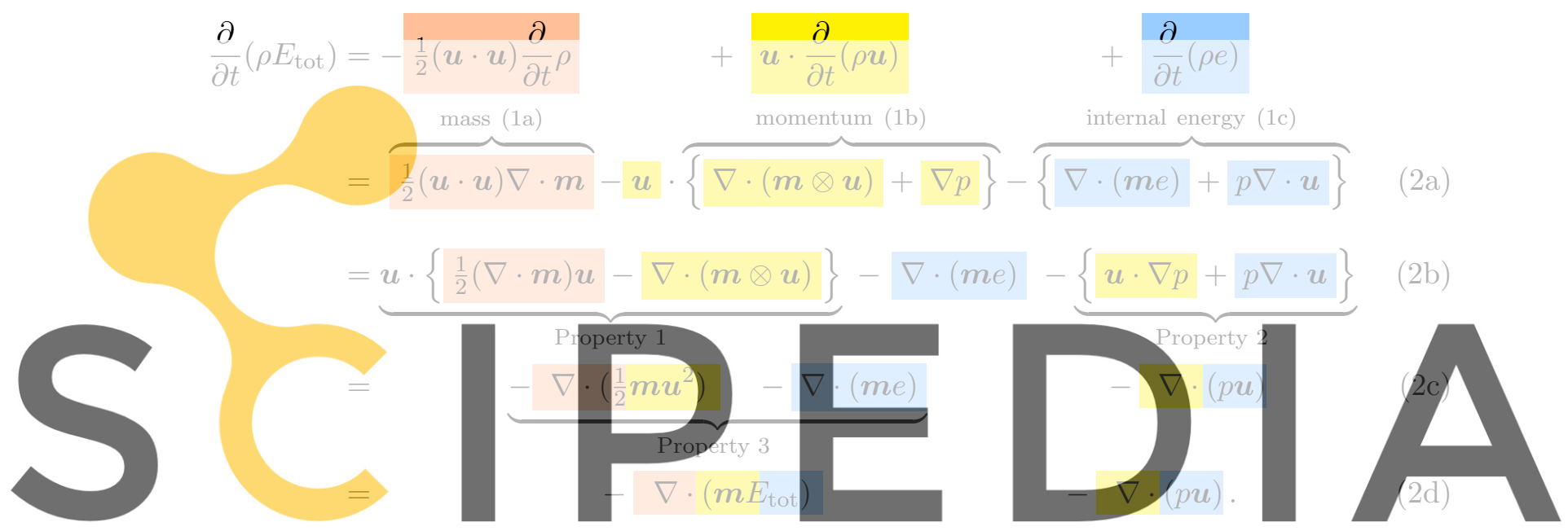

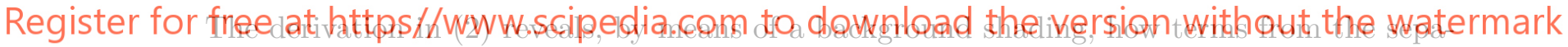
rate primary conservation laws have to be combined, requiring a certain level of compatibility. Analy tically this is not an issue, but in a discrete setting it is not straightforward. In the last two steps, from (2b) to (2c) and from (2c) to (2d), three analytic properties between the operators are essential. We will discuss these steps in detail, making a distinction between the various appearances of the $\nabla$-operator, indicated by subscripts.

Property $1((2 \mathrm{~b}) \rightarrow(2 \mathrm{c}))$. The convection operator for momentum conservation $\nabla_{\text {mom_conv }}$ together with the divergence operator of mass conservation $\nabla_{\text {mass }}$ form a convective divergence expression with operator $\nabla_{\text {toten_conv. }}$. This requires that (for any $\boldsymbol{m}$ ) the operator

$$
\mathcal{A}: \boldsymbol{u} \rightarrow \nabla_{\text {mom_conv }} \cdot(\boldsymbol{m} \otimes \boldsymbol{u})-\frac{1}{2}\left(\nabla_{\text {mass_div }} \cdot \boldsymbol{m}\right) \boldsymbol{u} \text { is skew symmetric. }
$$

Explanation. First, let the $L_{2}$-inner product for real-valued functions be defined through $((\phi, \psi)) \equiv$ $\int_{\Omega} \phi \psi \mathrm{d} \Omega$. Then, if an expression $\phi \mathcal{A} \phi$ can be rewritten as $\phi \mathcal{A} \phi \equiv \nabla \mathcal{B}(\phi)$ for some function $\mathcal{B}$, then (for all real-valued $\phi)((\phi, \mathcal{A} \phi))=\int_{\Omega} \phi \mathcal{A} \phi \mathrm{d} \Omega=\int_{\Omega} \nabla \mathcal{B}(\phi) \mathrm{d} \Omega=0$ because of Gauss' theorem and our assumption that the outer boundaries of $\Omega$ do not contribute. That means that $\mathcal{A}$ is skew-symmetric 
with respect to this $L_{2}$-inner product. Indeed, we can rewrite (for any $\boldsymbol{m}$ and $\left.\phi\right) \nabla \cdot(\boldsymbol{m} \phi)-\frac{1}{2}(\nabla \cdot \boldsymbol{m}) \phi \equiv$ $\frac{1}{2} \nabla \cdot(\boldsymbol{m} \phi)+\frac{1}{2}(\boldsymbol{m} \cdot \nabla) \phi$, which reveals the skew-symmetry as an operator acting on $\phi$.

Property $2((2 \mathrm{~b}) \rightarrow(2 \mathrm{c}))$. The gradient $\nabla_{\text {mom_grad }}$ is the negative $L_{2}$-transpose of the divergence $\nabla_{\text {inten_div }}$ in the dilatation term of the internal energy equation:

$$
\left(\left(\boldsymbol{u}, \nabla_{\text {mom_grad }} p\right)\right)=-\left(\left(\nabla_{\text {inten_div }} \cdot \boldsymbol{u}, p\right)\right) \text { for all } \boldsymbol{u} \text { and } p .
$$

In short hand, this property can be written as

$$
\nabla_{\text {mom_grad }}=-\nabla_{\text {inten_div }}^{T}\left(=-\nabla_{\text {mass }}^{T}\right) .
$$

Between parentheses the incompressible limit is given [34].

Property $3((2 c) \rightarrow(2 \mathrm{~d}))$. The convective operator $\nabla_{\text {inten_conv }}$ in the internal energy equation is the same as the divergence operator $\nabla_{\text {mom_conv }}$ in the momentum equation:

$$
\nabla_{\text {inten_conv }}=\nabla_{\text {mom_conv }}\left(\equiv \nabla_{\text {toten_conv }}\right) \text {. }
$$

The above properties reveal a close relation between the operators from the individual conservation laws. We will transfer these analytic properties towards the discrete setting.
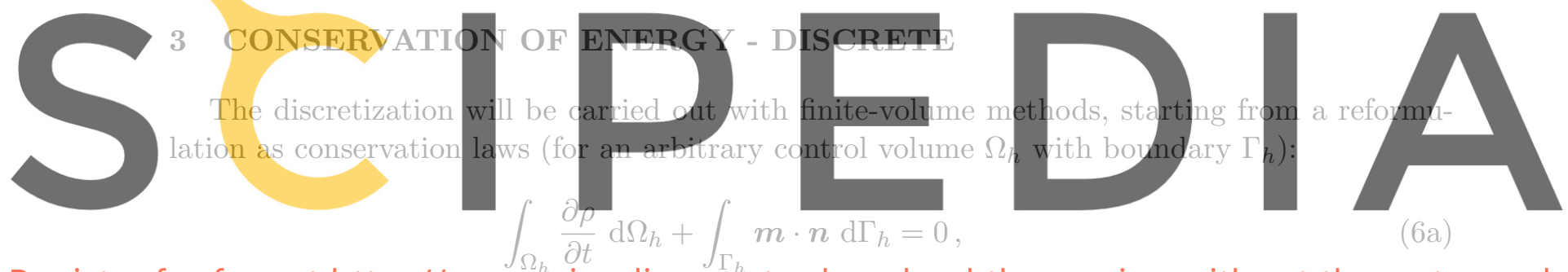

(6a)

Register for free at https//www.scipedia.com to download the version without the watermark

$$
\begin{aligned}
& \int_{\Omega_{h}} \frac{\partial \rho u}{\partial t} \mathrm{~d} \Omega_{h}+\int_{\Gamma_{h}}(m \cdot n) u \mathrm{~d} \Gamma_{h}=-\int_{\Gamma_{h}} p n \mathrm{~d} \Gamma_{h}, \\
& \int_{\Omega_{h}} \frac{\partial \rho e}{\partial t} \mathrm{~d} \Omega_{h}+\int_{\Gamma_{h}}(\boldsymbol{m} \cdot \boldsymbol{n}) e \mathrm{~d} \Gamma_{h}=-\int_{\Omega_{h}} p \nabla \cdot \boldsymbol{u} \mathrm{d} \Omega_{h} .
\end{aligned}
$$

The discretized versions of (6a)-(6c) will be abbreviated as

$$
\mathfrak{H} \frac{\partial \rho}{\partial t}+\mathfrak{D}_{\text {mass }} \boldsymbol{m}=0 ; \quad \mathfrak{H} \frac{\partial \rho \boldsymbol{u}}{\partial t}+\mathfrak{C}_{\text {mom }}^{\boldsymbol{m}} \boldsymbol{u}=-\mathfrak{G}_{\text {mom }} p ; \quad \mathfrak{H} \frac{\partial \rho e}{\partial t}+\mathfrak{C}_{\text {inten }}^{m} e=-p \mathfrak{D}_{\text {inten }} \boldsymbol{u} .
$$

Here, $\mathfrak{H}$ is a diagonal (matrix) operator containing the control volumes $\Omega_{h}$. The dependent variables are discrete (vector) grid functions, with the same (lower case) symbols as in the continuous case. The $\mathfrak{F} \mathfrak{r a k t u r}$-font operators denote volume-consistent $[17,32]$ approximations.

- $\mathfrak{D}_{\text {mass }}$ is a discrete divergence operator acting on the mass flux $\boldsymbol{m}$ in (6a). With the grid vector $\mathfrak{D}_{\text {mass }} \boldsymbol{m}$, a diagonal grid matrix $\operatorname{diag}\left(\mathfrak{D}_{\text {mass }} \boldsymbol{m}\right)$ can be formed. 
- $\mathfrak{C}_{\text {mom }}^{\boldsymbol{m}}$ is a discrete grid operator, acting on $\boldsymbol{u}$, for the convective term in the momentum equation (6b). Its coefficients depend on the mass flux $\boldsymbol{m}$.

$-\mathfrak{G}_{\text {mom }}$ is a discrete gradient operator in $(6 \mathrm{~b})$ acting on the pressure $p$.

- $\mathfrak{C}_{\text {inten }}^{m}$ is a discrete grid operator for the convective term in the conservation law for internal energy (6c), acting on $e$ and dependent on $\boldsymbol{m}$.

- $\mathfrak{D}_{\text {inten }}$ is a discrete divergence operator acting on the velocity $\boldsymbol{u}$ in (6c).

The sizes $\mathfrak{H}$ of the control volumes are included in the operators, i.e. the scaling in (7) is volume consistent $[17,32]$. This fits naturally in the finite-volume setting, and the symmetry properties of the discrete differential operators will come out more directly.

With the notation from (7), and similar to Eq. (2), the discrete (finite-volume) evolution of total energy can be formulated locally as

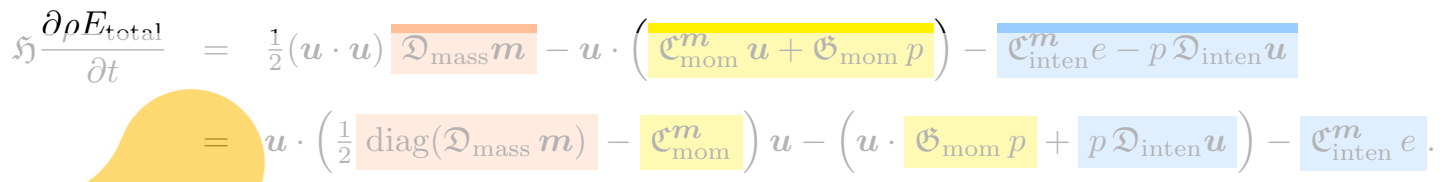

The last line in (8) corresponds with line (2b) in the analytic derivation. From here, we would like to make the steps to $(2 \mathrm{c})$ and $(2 \mathrm{~d})$ in this discrete version too. Therefore, let us find out which relations between the discrete operators have to be satisfied.
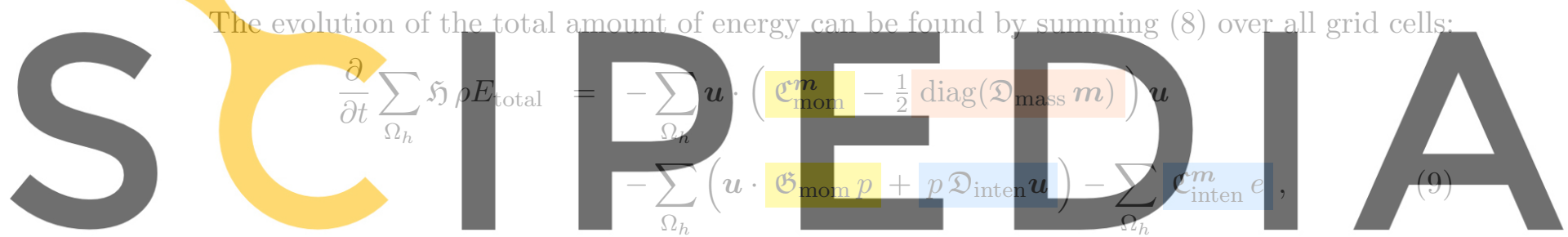

where on staggered grids the notation has to be interpreted with some liberty. Because of the

Register forifreelatnht: amount of energy in the domain. This volume-consistent [32] scaling property motivated us to 'hide' the size of the control volumes into the definition of the discrete operators.

The first two summations in the right-hand side of (7) are inner products in the space of scalar and vector-valued grid functions. The symmetry properties that we will discuss below are with respect to these inner products. We will see in the sequel that discrete energy conservation requires a certain amount of compatibility between the discrete operators.

Requirement 1. The first summation in the right-hand side of (9) should vanish, i.e. the matrix operator between the first pair of brackets should satisfy

$$
\mathfrak{C}_{\text {mom }}^{\boldsymbol{m}}-\frac{1}{2} \operatorname{diag}\left(\mathfrak{D}_{\text {mass }} \boldsymbol{m}\right) \text { is skew-symmetric. }
$$

This necessary and sufficient condition for discrete convective energy conservation has thus far been mentioned only a few times, e.g. $[8,23,24,35]$. It provides a relation between the diagonal of $\mathfrak{C}_{\text {mom }}^{m}$ and discrete mass conservation $\mathfrak{D}_{\text {mass }} \boldsymbol{m}$. 
Requirement 2. For the second sum in the right-hand side of (9) to vanish, the (pressure) gradient and the dilatational divergence should be each other's negative transpose:

$$
\mathfrak{G}_{\text {mom }}=-\mathfrak{D}_{\text {inten }}^{T}\left(=-\mathfrak{D}_{\text {mass }}^{T}\right) \text {. }
$$

In (11), between parentheses corresponds the incompressible limit [34]. It leads to a symmetric negative-definite Laplacian in the pressure Poisson equation. Condition (11) is necessary and sufficient for the contribution of the pressure to the total energy to vanish.

Finally, the discretization of the equation for internal energy, first of all, should be conservative. Additionally, for low Mach numbers [34] we would like it to approach a discretization for incompressible flow. This requires further consistency between the discrete operators.

Requirement 3. (A) To combine the momentum and internal-energy equations into a unified equation for total energy, the respective discrete convective operators should be the same:

$$
\mathfrak{C}_{\text {inten }}^{m}=\mathfrak{C}_{\mathrm{mom}}^{\mathrm{m}} \text {. }
$$

(B) A smooth transition from compressible flow to incompressible flow requires that the divergence operators in (6c) are consistent (in the incompressible limit) with the divergence in (6a):

$$
\mathfrak{D}_{\text {inten }}=\mathfrak{D}_{\text {mass }} \quad \vee \quad \mathfrak{C}_{\text {inten }}^{m} \rightarrow \rho_{0} \mathfrak{D}_{\text {mass }} u
$$

( $\rho_{0}$ is the incompressible density).

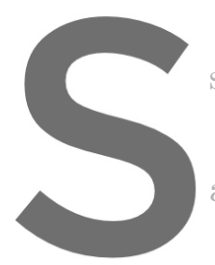

In view of the relations

satisfied.

A discretization satis
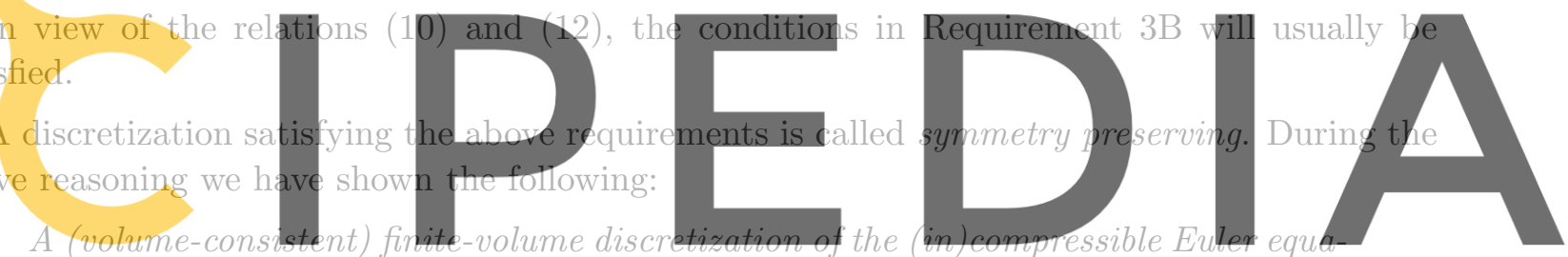

tions (1) preserves discrete energy if and only if it is symmetry-preserving.

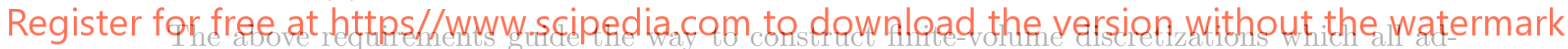

ditionally conserve discrete kinetic energy. In particular, the choice for the discretization of the convective term $\mathfrak{C}_{\mathrm{mom}}^{\mathrm{m}}$ induces all other discretizations:

1. Through Requirement 1 the discretization of the conservation of mass $\mathfrak{D}_{\text {mass }}$ is determined.

2. Requirement 2 then determines the discrete pressure gradient $\mathfrak{G}_{\text {mom }}$ in the conservation of momentum, and the dilatational divergence $\mathfrak{D}_{\text {inten }}$ in the conservation of internal energy.

3. Finally, Requirement 3 determines the discrete convective term $\mathfrak{C}_{\text {inten }}^{m}$ in the conservation of internal energy.

It is remarked that the above requirements have been derived starting from the symbolic discrete formulation in (7). As a consequence, all requirements for discrete energy conservation hold for any discretization method of the form (7) with a volume-consistent scaling, independent of the analytic formulation of the flow equations (for an overview, see [2]).

A diffusive term can be added, independently of the above discretizations. Perot [36] gives guide lines for arbitrary grids. 


\section{COMPRESSIBLE FLOW - COLLOCATED GRID}

On a structured collocated grid all flow variables are defined in 'cell centers' with a liberal interpretation of the meaning of 'center' (centroid, circumcenter, ...); see Fig. 1 (left). E.g., positioning the faces halfway the locations where the flow variables are defined (known as a Voronoi grid) is a valid option, as in Fig. 1 (right) of an unstructured grid.
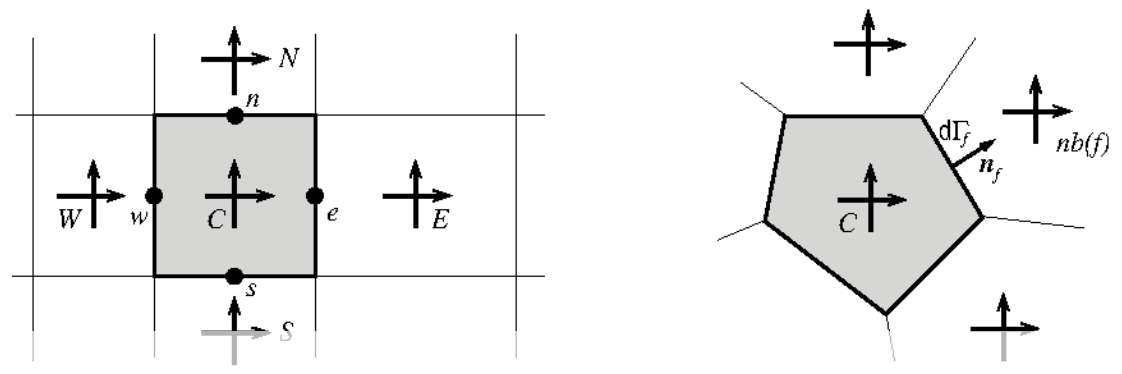

Figure 1: Control volumes for collocated grids: (left) structured with $\boldsymbol{u}$-locations halfway faces (= cellcentered); and (right) unstructured with faces halfway $\boldsymbol{u}$-locations (= face-or vertex-centered).

\subsection{Conservation of mass}
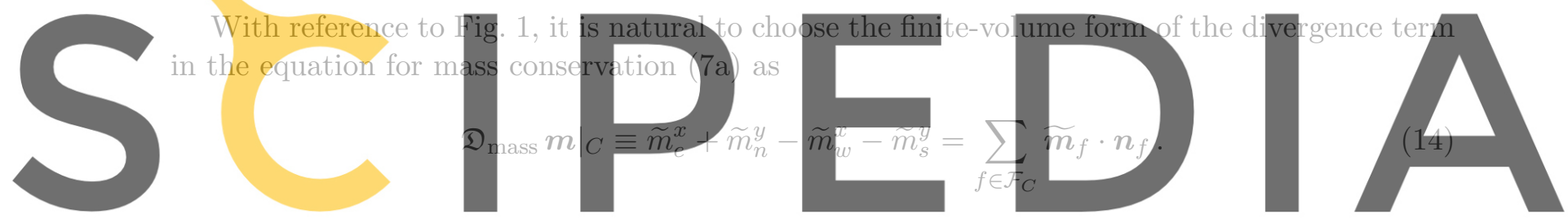

The summation is over the faces $f$ of the volume around $C$, together constituting the set $\mathcal{F}_{C}$,

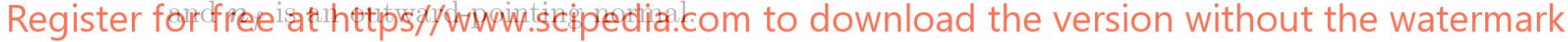

\subsection{Conservation of momentum}

Convection With similar notation, the discrete convective contribution to the momentum equation reads

$$
\left.\mathfrak{C}_{\mathrm{mom}}^{\boldsymbol{m}} \boldsymbol{u}\right|_{C} \equiv \widetilde{m}_{e}^{x} \boldsymbol{u}_{e}+\widetilde{m}_{n}^{y} \boldsymbol{u}_{n}-\widetilde{m}_{w}^{x} \boldsymbol{u}_{w}-\widetilde{m}_{s}^{y} \boldsymbol{u}_{s}=\sum_{f \in \mathcal{F}_{C}}\left(\widetilde{\boldsymbol{m}}_{f} \cdot \boldsymbol{n}_{f}\right) \boldsymbol{u}_{f}
$$

To compute the fluxes at the cell faces, an equal-weighted $\frac{1}{2}-\frac{1}{2}$ interpolation for the velocity component $u$ must be applied:

$$
\boldsymbol{u}_{f}=\frac{1}{2}\left(\boldsymbol{u}_{C}+\boldsymbol{u}_{n b(f)}\right),
$$

where $n b(f)$ denotes the neighboring grid cell sharing the face $f$. In this way, the coefficients in the convective contribution are skew-symmetric outside the diagonal. The $\frac{1}{2}-\frac{1}{2}$ interpolation is essential, even when the edges are not half-way between the cell centers. Jameson [22,37], 
interpreted the values in the cell centers as averages over the cells, after which a $\frac{1}{2}-\frac{1}{2}$ averaging at the separating face is natural.

With the interpolation (16), the coefficient on the diagonal of $\mathfrak{C}_{\mathrm{mom}}^{m}(15)$ becomes

$$
\operatorname{diag}\left(\mathfrak{C}_{\text {mom }}^{\boldsymbol{m}}\right)=\frac{1}{2} \sum_{f \in \mathcal{F}_{C}}\left(\widetilde{\boldsymbol{m}}_{f} \cdot \boldsymbol{n}_{f}\right) \stackrel{(14)}{=} \frac{1}{2} \mathfrak{D}_{\text {mass }} \boldsymbol{m} .
$$

Hence the vector $\operatorname{diag}\left(\mathfrak{C}_{\text {mom }}^{\boldsymbol{m}}\right)-\frac{1}{2} \mathfrak{D}_{\text {mass }} \boldsymbol{m}$ vanishes. In fact, the latter requirement determines the choice of $\mathfrak{D}_{\text {mass }}$ ! The above discretization, (14) + (15) with interpolation (16), satisfies the main Requirement 1: $\mathfrak{C}_{\text {mom }}^{\boldsymbol{m}}-\frac{1}{2} \operatorname{diag}\left(\mathfrak{D}_{\text {mass }} \boldsymbol{m}\right)$ is skew symmetric, for all choices of the mass fluxes $\widetilde{m}$. There is some freedom left to use geometry information to interpolate from the values of $\boldsymbol{m}$ in the cell centers to the values of $\widetilde{m}$ at the faces [38].

Pressure gradient A natural choice for the finite-volume form of the pressure gradient is

$$
\left.\mathfrak{G}_{\operatorname{mom}} p\right|_{C} \equiv\left(\tilde{p}_{e}-\tilde{p}_{w}\right) e_{x}+\left(\widetilde{p}_{n}-\tilde{p}_{s}\right) e_{y}=\sum_{f \in \mathcal{F}_{C}} \tilde{p}_{f} n_{f} .
$$

Once more using equal-weighted interpolation, as in (16), we define the pressure 'fluxes' as $\tilde{p}_{f}=\frac{1}{2}\left(p_{C}+p_{n b(f)}\right)\left|\mathrm{d} \Gamma_{f}\right|$. The gradient operator can now be rewritten as

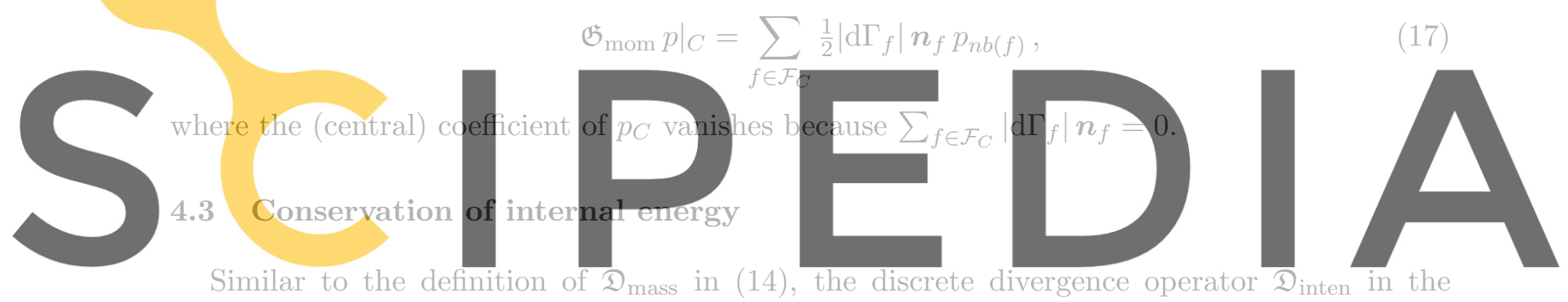

Similar to the definition of $\mathfrak{D}_{\text {mass }}$ in (14), the discrete divergence operator $\mathfrak{D}_{\text {inten }}$ in the Register for free at https//www.Scipedia.com to download the version without the watermark Dinten $\left.u\right|_{C} \equiv \widetilde{u}_{e}^{x}+\widetilde{u}_{n}^{y}-\widetilde{u}_{w}^{x}-\widetilde{u}_{s}^{y}=\sum_{f \in \mathcal{F}_{C}} \widetilde{u}_{f} \cdot n_{f}$.

Again, equal-weighted $\left(\frac{1}{2}-\frac{1}{2}\right)$ interpolation is used to define the face fluxes: $\widetilde{\boldsymbol{u}}_{f}=\frac{1}{2}\left(\boldsymbol{u}_{C}+\right.$ $\left.\boldsymbol{u}_{n b(f)}\right)\left|\mathrm{d} \Gamma_{f}\right|$. The divergence operator can now be rewritten as

$$
\left.\mathfrak{D}_{\text {inten }} \boldsymbol{u}\right|_{C}=\sum_{f \in \mathcal{F}_{C}} \frac{1}{2}\left|\mathrm{~d} \Gamma_{f}\right| \boldsymbol{n}_{f} \cdot \boldsymbol{u}_{n b(f)},
$$

where the (central) coefficient of $\boldsymbol{u}_{C}$ has vanished as in (17). Comparing this operator with the gradient from (17), it can be seen that $\mathfrak{D}_{\text {inten }}=\mathfrak{D}_{\text {mass }}$ and $\mathfrak{G}_{\text {mom }}$ are each other's negative transpose, as imposed by Requirement 2 .

The convective term in the equation for internal energy reads

$$
\left.\mathfrak{C}_{\text {inten }}^{\boldsymbol{m}} e\right|_{C} \equiv \widetilde{m}_{e}^{x} e_{e}+\widetilde{m}_{n}^{y} e_{n}-\widetilde{m}_{w}^{x} e_{w}-\widetilde{m}_{s}^{y} e_{s}=\sum_{f \in \mathcal{F}_{C}}\left(\widetilde{\boldsymbol{m}}_{f} \cdot \boldsymbol{n}_{f}\right) e_{f},
$$

which has the same structure as (15) (cf. Requirement 3). 


\subsection{Local conservation of total energy}

Satisfying the Requirements 1-3 guarantees global conservation of total energy. For finitevolume discretizations also local energy conservation follows. The local energy flux can be found by substituting the fluxes given in (15), (17), (18) and (19) into the discrete evolution of total energy (8). For the above example, this results in a local energy flux given by $\left[\frac{1}{2} \widetilde{\boldsymbol{m}}_{f}\left(\boldsymbol{u}_{C} \cdot \boldsymbol{u}_{n b(f)}\right)+\right.$ $\left.\frac{1}{2}\left(p_{n b(f)} \boldsymbol{u}_{C}+p_{C} \boldsymbol{u}_{n b(f)}\right)+\widetilde{\boldsymbol{m}}_{f} e_{f}\right] \cdot \boldsymbol{n}_{f}$.

\section{DISCUSSION}

We have unraveled a strategy to derive supra-conservative finite-volume discretizations for compressible Euler flow that possess additional discrete (secondary) conservation properties for energy. Mimicking the analytic derivation, and following a principle of non-interference, the key ingredient is a close consistency between the discrete momentum equation and the discrete mass equation (Requirement 1). In particular, the diagonal of the discrete convection operator directly determines the discrete divergence in the mass equation and in the dilatation term of the internal energy equation (Requirement 3). Also, it determines the discrete pressure gradient (Requirement 2). An equal-weighted interpolation for the velocity from cell centers to cell faces is essential to achieve the required compatibility, irrespective of any stretching of the grid. More details can be found in a forthcoming publication [39].

One 'reward' for $t$. without needing any lower bound, as in the in compressible case, but in is that subtleties in dissipation $[31,38]$.
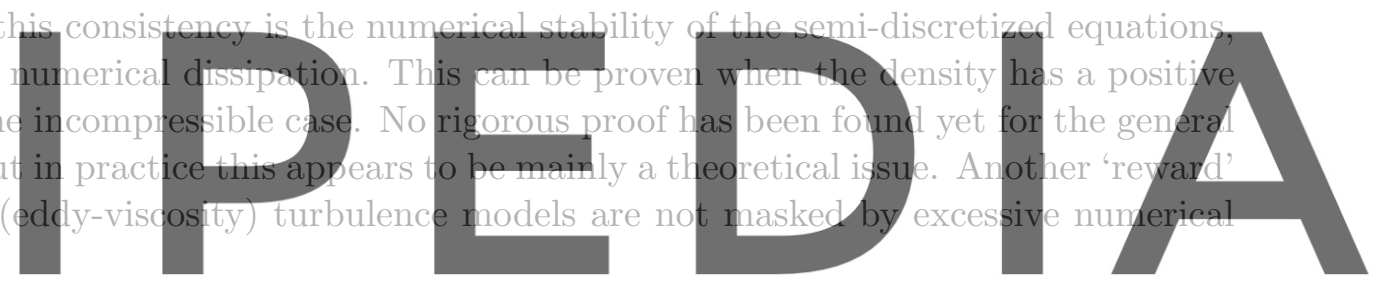

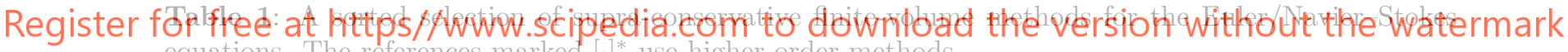
equations. The references marked [.] ${ }^{*}$ use higher-order methods.

\begin{tabular}{llll} 
flow & grid & staggered & collocated \\
\hline \hline \multirow{2}{*}{ incompressible } & \multirow{2}{*}{ structured } & {$[35]$,} & {$[4,-13]^{*}$} \\
\cline { 2 - 4 } & & {$[8,11]$} \\
\cline { 2 - 4 } & unstructured & {$[15,41,42]$} & {$[14,15]$} \\
\hline \multirow{2}{*}{ compressible } & \multirow{2}{*}{ structured } & {$[24]$} & {$[1,21,22,28]$,} \\
& & {$[23,25,31]^{*}$}
\end{tabular}

To demonstrate the performance of the above methods, we point the reader to a number of papers that are successfully using them. Table 1 gives several of such supra-conservative finite-volume methods for the Euler and/or Navier-Stokes equations. The table has been sorted according to the grid used (structured or unstructured) and the positioning of the unknowns (staggered or collocated). Also, higher-order $(>2)$ variants have been indicated.

Figure 2 shows an example from [31] of an energy-preserving discretization of compressible flow past a delta wing at a chord Reynolds number $R e_{c}=150,000$, featuring a natural transition 

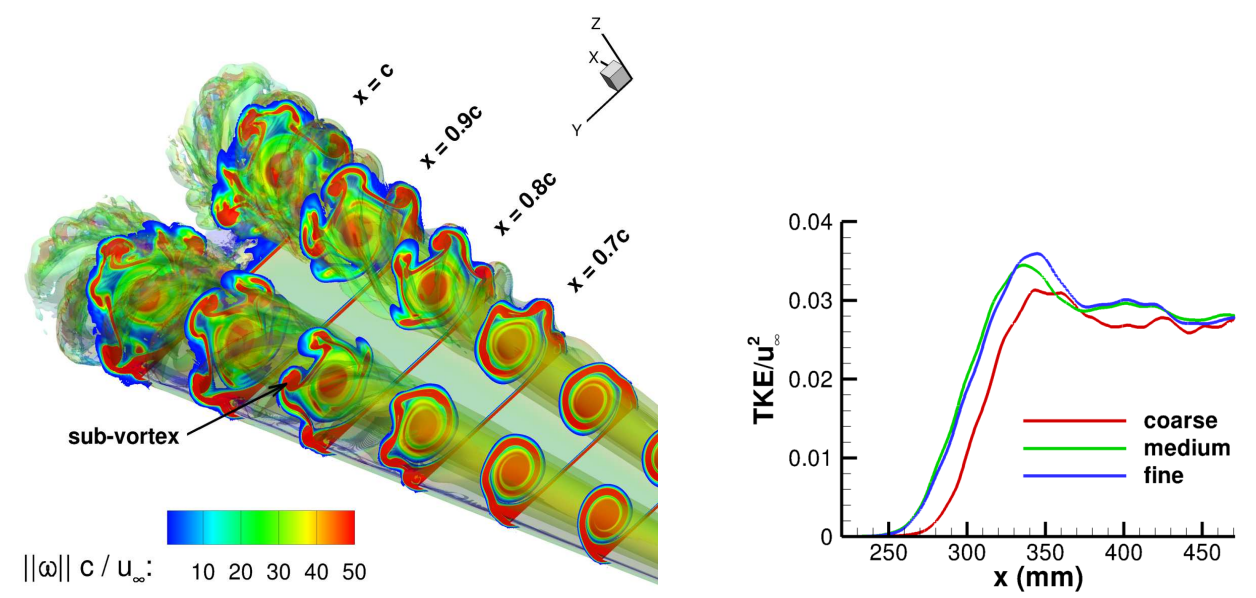

Figure 2: Numerical simulation of transitional flow nast a delta wing at $R e_{c}=150,000$. (left) Axial slices of the instantaneous vorticity magnitude. (right) Turbulent kinetic energy along a chord line.

from laminar to turbulent flow. The left-hand graph reveals the vortical structures above the wing showing the transition. The right-hand graph gives the turbulent kinetic energy, on three grids, in streamwise direction. It shows the start of the transition at $x=250 \mathrm{~mm}$, corresponding with a local Reynolds number around $R e_{x}=80,000$. This is in close agreement with the experimentally found start of transition at $R e_{x} \approx 78,000$ [43]. The good correspondence shows

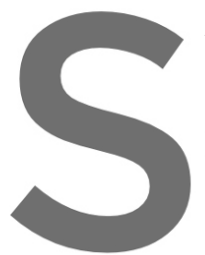

the potential of discretiz

REFERENCES
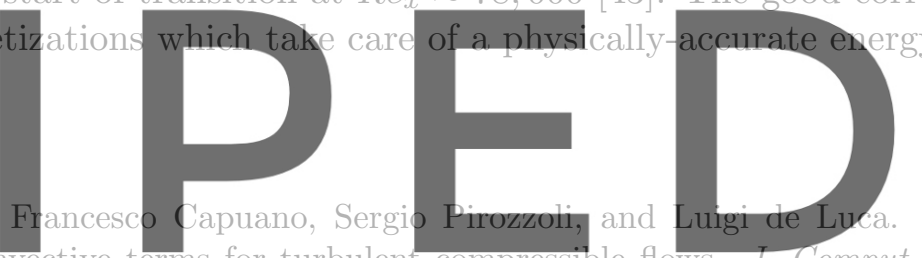

formulations of convective terms for turbulent

\section{Register for free at https/Awww.scipedia.com to download the version without the watermark} ical solution of the Navier-Stokes equations. Appl. Mech. Rev., 71:010803-1 - 010803-19, 2019. httips://doi.org/10.1115/1.4042820.

[3] N. A. Phillips. An example of non-linear computational instability. In The Atmosphere and the Sea in Motion, pages 501-504. The Rockefeller Institute Press, 1959.

[4] A. Arakawa. Computational design for long-term numerical integration of the equations of fluid motion: Two-dimensional incompressible flow. Part I. J. Comput. Phys., 1:119-143, 1966.

[5] S. A. Piacsek and G. P. Williams. Conservation properties of convection difference schemes. J. Comput. Phys., 6:392-405, 1970.

[6] Y. Morinishi, T. S. Lund, O. V. Vasilyev, and P. Moin. Fully conservative higher order finite difference schemes for incompressible flow. J. Comput. Phys., 143:90-124, 1998.

[7] O. V. Vasilyev. High order difference schemes on non-uniform meshes with good conservation properties. J. Comput. Phys., 157:746-761, 2000.

[8] Bas van't Hof and Mathea J. Vuik. Symmetry-preserving finite-difference discretizations of arbitrary order on structured curvilinear staggered grids. J. Comput. Sci., 36:101008, 2019. 
[9] Magnus Svärd and Jan Nordström. Review of summation-by-parts schemes for initial-boundaryvalue problems. J. Comput. Phys., 268:17-38, 2014.

[10] A. E. P. Veldman and K. Rinzema. Playing with nonuniform grids. J. Eng. Math., 26:119-130, 1991.

[11] R. W. C. P. Verstappen and A. E. P. Veldman. Direct numerical simulation of turbulence at lesser costs. J. Eng. Math., 32:143-159, 1997.

[12] R. W. C. P. Verstappen and A. E. P. Veldman. Spectro-consistent discretization: a challenge to RANS and LES. J. Eng. Math., 34:163-179, 1998.

[13] R. W. C. P. Verstappen and A. E. P. Veldman. Symmetry-preserving discretization of turbulent flow. J. Comput. Phys., 187:343-368, 2003.

[14] F. X. Trias, O. Lehmkuhl, A. Oliva, C. D. Pérez-Segarra, and R. W. C. P. Verstappen. Symmetrypreserving discretization of Navier-Stokes equations on collocated unstructured grids. J. Comput. Phys., 258:246-267, 2014.

[15] Lluís Jofre, Oriol Lehmkuhl, Jordi Ventosa, F Xavier Trias, and Assensi Oliva. Conservation properties of unstructured finite-volume mesh schemes for the Navier-Stokes equations. Numer. Heat Transfer, Part B: Fundamentals, 65(1):53-79, 2014.

[16] W. J. Feiereisen, W. C. Reynolds, and J. H. Ferziger. Numerical simulation of a compressible, homogeneous, turbulent shear flow. Report TF-13, Thermosciences Division, Mechanical Engineering, Stanford University, 1981.

[17] E. J. Caramana, D. E. Burton, M. J. Shashkov, and P. P. Whalen. The construction of compatible hydrodynamics algorithms utilizing conservation of total energy. J. Comput. Phys., 146(1):227-262, 1998.

[18] S. Pirozzoli. Stabilized non-dissipative approximations of Euler equations in generalized curvilinear coordinates. J. Comput. Phys., 230:2997-3014, 2011.

[19] A. E. Honein and P. Moin. Higher entropy conservation and numerical stability of compressible turbulence simulations. J. Comput. Phys., 201:531-545, 2004.

[20] Praveen Chandrashekar. Kinetic energy preserving and entropy stable finite volume schemes for compressible Euler and Navier-Stokes equations. Commun. Comput. Phys., 14(5):1252-1286, 2013.

[21] F. Ducros, F. Laporte, T. Souleres, V. Guinot, P. Moinat, and B. Caruelle. High-order fluxes for conservative skew-symmetric-like schemes in structured meshes: application to compressible flows. J. Comput. Phys., 161(1):114-139, 2000.

[22] A. Jameson. Formulation of kinetic energy preserving conservative schemes for gas dynamics and direct numerical simulation of one-dimensional viscous compressible flow in a shock tube using entropy and kinetic energy preserving schemes. J. Sci. Comput., 34:188-208, 2008.

[23] J. C. Kok. A high-order low-dispersion symmetry-preserving finite-volume method for compressible flow on curvilinear grids. J. Comput. Phys., 228:6811-6832, 2009.

[24] Y. Morinishi. Skew-symmetric form of convective terms and fully conservative finite difference schemes for variable density low-Mach number flows. J. Comput. Phys., 229:276-300, 2010.

[25] W. Rozema, J. C. Kok, R. W. C. P. Verstappen, and A. E. P. Veldman. A symmetry-preserving discretisation and regularisation model for compressible flow with application to turbulent channel flow. J. Turbul., 15(6):386-410, 2014.

[26] B. Sanderse. Energy-conserving Runge-Kutta methods for the incompressible Navier-Stokes equations. J. Comput. Phys., 233:100-131, 2013. 
[27] Francesco Capuano, Gennaro Coppola, Luis Rández, and Luigi de Luca. Explicit Runge-Kutta schemes for incompressible flow with improved energy-conservation properties. J. Comput. Phys., 328:86-94, 2017.

[28] P. K. Subbareddy and G. V. Candler. A fully discrete, kinetic energy consistent finite-volume scheme for compressible flows. J. Comput. Phys., 228:1347-1364, 2009.

[29] W. Rozema, H. J. Bae, P. Moin, and R. Verstappen. Minimum-dissipation models for large-eddy simulation. Phys. Fluids, 27:085107, 2015.

[30] J. Reiss. A family of energy stable, skew-symmetric finite difference schemes on collocated grids. J. Sci. Comput., 65:1-18, 2015.

[31] Wybe Rozema, Johan C. Kok, Arthur E. P. Veldman, and Roel W. C. P. Verstappen. Numerical simulation with low artificial dissipation of transitional flow over a delta wing. J. Comput. Phys., 405:109182, 15 March 2020.

[32] Arthur E. P. Veldman. A general condition for kinetic-energy preserving discretization of flow transport equations. J. Comput. Phys., 398:108894, 2019.

[33] Arthur E. P. Veldman. 'Missing' boundary conditions? Discretize first, substitute next, and combine later. SIAM J. Sci. Stat. Comput., 11(1):82-91, 1990.

[34] Rupert Klein. Semi-implicit extension of a Godunov-type scheme based on low Mach number asymptotics I: One-dimensional flow. J. Comput. Phys., 121(2):213-237, 1995.

[35] B. van't Hof and A. E. P. Veldman. Mass, momentum and energy conserving (MaMEC) discretizations on general grids for the compressible euler and shallow water equations. J. Comput. Phys., 231:4723-4744, 2012.

[36] J. Blair Perot and V. Subramanian. Discrete calculus methods for diffusion. J. Comput. Phys., 224(1):59-81, 2007.

[37] A. Jameson, W. Schmidt, and E. Turkel. Numerical solution of the Euler equations by finite volume methods using Runge-Kutta time stepping schemes. AIAA Paper 81-1259, 1981.

[38] Wybe Rozema, Roel W. C. P. Verstappen, Johan C. Kok, and Arthur E. P. Veldman. Low-dissipation simulation methods and models for turbulent subsonic flow. Arch. Comput. Meth. Eng., 27(1):299330, 2020. DOI: 10.1007/s11831-018-09307-7.

[39] Arthur E. P. Veldman. Supra-conservative finite-volume methods for the Euler equations of subsonic compressible flow. SIAM Review, under revision, 2021.

[40] Frédéric N. Felten and Thomas S. Lund. Kinetic energy conservation issues associated with the collocated mesh scheme for incompressible flow. J. Comput. Phys., 215(2):465-484, 2006.

[41] B. Perot. Conservation properties of unstructured staggered mesh schemes. J. Comput. Phys., 159(1):58-89, 2000.

[42] Xing Zhang, David Schmidt, and Blair Perot. Accuracy and conservation properties of a threedimensional unstructured staggered mesh scheme for fluid dynamics. J. Comput. Phys., 175(2):764791, 2002.

[43] A. J. Riley and M. V. Lowson. Development of a three-dimensional free shear layer. J. Fluid Mech., 369:49-88, 1998. 